\title{
RAPID DETECTION OF ASIATICOSIDE BY IMMUNOCHROMATOGRAPHIC ASSAY
}

\section{(ㄷ) Sritularak B. ${ }^{1}$, Juengwatanatrakul-T. ${ }^{2}$, Putalun W. ${ }^{3}$, Tanaka H. ${ }^{4}$, Morimoto S. ${ }^{4}$}

${ }^{1}$ Faculty of Pharmaceutical Sciences, Chulalongkorn University, Bangkok, Thailand

${ }^{2}$ Faculty of Pharmaceutical Sciences, Ubon Ratchathani University, Ubon Ratchathani, 34190, Thailand

${ }^{3}$ Faculty of Pharmaceutical Sciences, Khon Kaen University, Khon Kaen, 40002, Thailand

${ }^{4}$ Graduate School of Pharmaceutical Sciences, Kyushu University, Fukuoka, Japan

Asiaticoside (AS), [6- $[[3,4-$ dihydroxy-6(hydroxymethyl)-5- (3,4,5-trihydroxy-6-methyloxan-2-yl)oxyoxan-2-yl] oxymethyl]-3,4,5-trihydroxyoxan-2-yl] 10,11-dihydroxy-9- (hydroxyme-thyl)-1,2,6a,6b,9,12a-hexamethyl-2,3,4,5,6,6a,7,8,8a,10,11, $12,13,14 \mathrm{~b}$-tetradecahydro- $1 \mathrm{H}$-picene-4a-carboxylate, has been identified as the most active compound in Centella asiatica (L.) urb. In order to screen large numbers of samples for the presence of AS, a rapid and simple technique is required for application to small quantities of test samples. Immunoassays using monoclonal antibodies could be useful for the determination of small quantities of AS in plant extracts (1). In this study, an immunochromatographic strip test has been developed for the detection of AS in plant samples using monoclonal antibody against AS (2). The qualitative assay was based on a competitive immunoassay in which the detector re- agent consisted of colloidal gold particles coated with the respective AS antibodies. The capture reagent was an AS-human serum albumin (HSA) conjugate immobilized onto a test strip membrane. The sample containing AS and the detection reagent were incubated with the immobilized capture reagent. The AS in the sample competed in binding to the limited amount of antibodies in the detection reagent with the immobilized ASHSA conjugates and, hence, positive samples showed no color in the capture spot zone. The detection limit for the strip test was $12.5 \mu \mathrm{g} / \mathrm{mL}$. The immunochromatographic strip test is useful as a rapid and simple screening method for the determination of small quantities of AS in plant extracts, body fluids and foods.

References: (1) Juengwatanatrakul T (2011) Analyst 136:1013-17. (2) Sritularak B (2012) J Nat Med 66:279-83.

\section{THROMBOLYTIC PROPERTIES OF MEDICAL LEECH EXTRACT}

\section{() Antipov A., Kamenev 0.}

Hiruline laboratory, Ltd, Saint-Petersburg, Russia, E-mail: info@hiruline.ru

The purpose of this study was to determine the thrombolytic activity of the extract of bred medical leeches and to compare it with modern thrombolytic substances. The following in vitro model was used: the clots were made from $100 \mu \mathrm{l}$ of rat blood drops. The clot formation time (CFT) was 1, 2, 4, 6 or 12 hours respectively. Every clot was placed in a cuvette of the spectrophotometer "Specord M-40" (Germany) with plasma at $37^{\circ} \mathrm{C}(\mathrm{ph} 7,4)$. The leech extract or one of the thrombolytic substances: Streptokinase, Urokinase (UPA), Alteplase (rt-PA), Lanoteplase (n-PA), were added at equal concentration of $1 \mathrm{mg} / \mathrm{ml}$. The content of cuvette was constantly stirred. The degree of clot lysis was measured by the hemoglobin concentration in plasma at $560 \mathrm{~nm}$ with a help of the calibration curve. The measurements were made in 10 minutes, 0.5,
$1.01 .5,3,6,9,12$ and 24 hours after adding of test substance. Each series for each CFT had 7 experiments for each substance. A control experiment with 1 hour clots was made without any substance. It showed that the hemoglobin is not released from the clot by itself. Statistical analysis was performed using STATISTICA 6 software (Statsoft Inc.). The results of the research showed that thrombolytic substances were more effective than leech extract during the 1 hour for 1 and 1,5 hour for 2 hour clots. After 3 hours the extract had a stronger thrombolytic effect for 1 hour clots. The advantages of leech extract were more expressed for 4-, 6-and 12 hour clots. The study demonstrates the presence and efficiency of thrombolytic activity of the extract of bred medical leeches. A thrombolytic agent of a new type can be created. 